\title{
Modelling the effects of ongoing alpha activity on visual perception: The oscillation-based probability of response
}

\author{
Agnese Zazio $^{\mathrm{a}, *}$, Marco Schreiber ${ }^{\mathrm{b}}$, Carlo Miniussi ${ }^{\mathrm{a}, \mathrm{c}}$, Marta Bortoletto ${ }^{\mathrm{a}}$ \\ ${ }^{\text {a }}$ Cognitive Neuroscience Section, IRCCS Istituto Centro San Giovanni di Dio Fatebenefratelli, Brescia, Italy \\ ${ }^{\mathrm{b}}$ Independent Researcher \\ ${ }^{\mathrm{c}}$ Center for Mind/Brain Sciences - CIMeC, University of Trento, Rovereto, Italy
}

\section{A R T I C L E I N F O}

\section{Keywords:}

Neural oscillations

Prestimulus

Alpha

Gamma

Perception

Psychometric function

Cross-Frequency

\begin{abstract}
A B S T R A C T
Substantial evidence has shown that ongoing neural activity significantly contributes to how the brain responds to upcoming stimuli. In visual perception, a considerable portion of trial-to-trial variability can be accounted for by prestimulus magneto/electroencephalographic (M/EEG) alpha oscillations, which play an inhibitory function by means of cross-frequency interactions with gamma-band oscillations. Despite the fundamental theories on the role of oscillations in perception and cognition, the current literature lacks a clear theorization of the neural mechanisms underlying the effects of prestimulus activity, including electrophysiological phenomena at different scales (e.g., local field potentials and macro-scale M/EEG). Here, we present a model called the oscillationbased probability of response (OPR), which directly assesses the link between meso-scale neural mechanisms, macro-scale M/EEG, and behavioural outcome. The OPR includes distinct meso-scale mechanisms through which alpha oscillations modulate M/EEG gamma activity, namely, by decreasing $a$ ) the amplitude and/or $b$ ) neural synchronization of gamma oscillations. Crucially, the OPR makes specific predictions on the effects of these mechanisms on visual perception, as assessed through the psychometric function.

significance statement: The oscillation-based probability of response (OPR) is grounded on a psychophysical approach focusing on the psychometric function estimation and may be highly informative in the study of ongoing brain activity, because it provides a tool for distinguishing different neural mechanisms of alpha-driven modulation of sensory processing.
\end{abstract}

\section{Introduction}

The response of neurons is well known to not merely depend on external input; indeed, a repeated presentation of the same stimulus gives rise to highly variable responses at the neural level as well as at the behavioural level (Arieli et al., 1996; Vogels et al., 1989). Interestingly, such response variability can be accounted for by fluctuations in ongoing brain activity, as revealed by invasive (Arieli et al., 1996) and non-invasive (Kayser et al., 2016; Weisz et al., 2014) electrophysiological recordings, functional magnetic resonance imaging (Baldassarre et al., 2012; Hesselmann et al., 2008a,) and behavioural (Song et al., 2014) studies.

The time-frequency pattern of brain oscillations represents a key feature by which ongoing activity shapes both neural and behavioural responses (Ai and Ro, 2014; Baumgarten et al., 2016; Haegens et al., 2011; Kayser et al., 2016; Leske et al., 2015; Linkenkaer-Hansen, 2004; Mazaheri et al., 2009; Schubert et al., 2008; van Dijk et al., 2008).
Specifically, ongoing oscillations within the alpha band (frequency range: $8-13 \mathrm{~Hz}$ ), as measured by magneto/electroencephalographic (M/EEG) recordings, represent a major factor in accounting for response variability in the domain of visual perception (Busch et al., 2009; Iemi et al., 2017; Iemi and Busch, 2018; Lange et al., 2014; Mathewson et al., 2009; van Dijk et al., 2008).

In recent decades, neuroscientists have developed fundamental theories highlighting the role of oscillations in brain dynamics, such as in neural inhibition (inhibition timing hypothesis, Klimesch et al., 2007; gating by inhibition, Jensen and Mazaheri, 2010) and neural communication (communication through coherence, Fries, 2005). Nevertheless, the micro- (e.g., single-unit measurements) and mesoscale (e.g., local field potential, LFP) neural mechanisms that contribute to M/EEG oscillatory activity and their link with behaviour are far from established (Cohen, 2017; Musall et al., 2014).

\footnotetext{
* Corresponding author at: Cognitive Neuroscience Section, IRCCS Istituto Centro San Giovanni di Dio Fatebenefratelli Via Pilastroni 4, 25125 Brescia Italy

E-mail address: agnese.zazio@cognitiveneuroscience.it (A. Zazio).
} 


\section{The oscillation-based probability of response}

The oscillation-based probability of response (OPR) is a model that fuses neurophysiological evidence, mathematical modelling and a psychophysical approach. The aim of the model is twofold: first, to identify potential neural mechanisms of alpha-gamma interactions that support the effects of prestimulus M/EEG oscillations on visual perception (Lange et al., 2014; Ruhnau et al., 2014; Sadaghiani and Kleinschmidt, 2016; Zoefel and VanRullen, 2017); second, to provide a framework with clear predictions on the effects on perception, so that behavioural performance can be exploited to distinguish the underlying neural mechanisms that cannot be disentangled by means of M/EEG.

The OPR is based on the following key points: 1) alpha oscillations affect visual perception, 2) alpha activity plays an inhibitory role, 3) alpha inhibition occurs through alpha-gamma cross-frequency interactions, 4) alpha-modulated gamma oscillations affect the probability of neurons in sensory areas to respond to an incoming stimulus; and 5) the probability of response to incoming stimuli is specifically modulated by distinct coupling mechanisms of alpha-gamma interaction.

Crucially, the OPR includes the estimation of the response probability for a wide range of stimulus intensities and therefore generates a psychometric function. In this framework, the estimated psychometric function represents an extremely powerful tool because it provides suggestions for the involvement of two distinct neural mechanisms, which cannot to be discerned non-invasively (Cohen, 2017; Whittingstall and Logothetis, 2009). We therefore propose the application of OPR to generate hypotheses on distinct mechanisms involved in alpha-driven modulation of sensory processing, based on the specific modification of the psychometric function.

\subsection{Alpha oscillations affect visual perception}

Oscillatory activity within the alpha band is dominant in the human brain; it represents the strongest electrophysiological signal that can be measured non-invasively and is observed transversely across cognitive domains (Berger, 1929; Klimesch, 2012).

The effect of spontaneous variations in M/EEG alpha activity on perception is well documented. Typically, to detect the effects of such spontaneous fluctuations, studies employ stimuli at the individual sensory threshold, i.e., the so-called "near-threshold stimuli", by definition detected in half of the trials (for a review see Ruhnau et al., 2014). Single-trial analysis is then performed to assess within-subjects variance (Pernet et al., 2011).

Experimental evidence has shown that perceptual performance in the visual domain is affected by prestimulus M/EEG alpha power. Specifically, trials with a low alpha power preceding stimulus onset lead to a higher probability of stimulus detection, both between and within subjects (Fig. 1A; Busch and Van Rullen, 2010; Hanslmayr et al., 2007; van Dijk et al., 2008), and it has recently been suggested that this may be due to a more liberal criterion in the response rather than an improved perceptual acuity (Iemi et al., 2017; Limbach and Corballis, 2016). Furthermore, a few studies reported a difference in the visual detection rate between opposite phases of 7-10 Hz oscillations (Fig. 1B; Busch et al., 2009; Busch and Van Rullen, 2010; Mathewson et al., 2009; but see Benwell et al., 2017,). Consistently, ongoing M/ EEG alpha power and phase have been shown to be relevant factors in predicting transcranial magnetic stimulation-induced phosphene perception (Dugué et al., 2011; Romei et al., 2008).

Moreover, a few studies have provided evidence of a causal role of prestimulus alpha oscillations on perception by modulating the alpha rhythm. A traditional way to modulate alpha power is by means of spatial attention: alpha power is lower for the attended than for the unattended visual hemifield (Worden et al., 2000; Sauseng et al., 2005), thereby affecting detection performance (Busch and Van Rullen, 2010). More recently, alpha oscillations have been modulated with entrainment mechanisms, reflecting the phase alignment of the brain's oscillatory activity to external rhythmic stimulation by sensory stimulation (e.g., visual or auditory rhythmic stimuli; Spaak et al., 2014; Henry and Obleser, 2012) and through non-invasive brain stimulation techniques (Thut et al., 2017, 2011; Helfrich et al., 2016). Entrainment of an endogenous alpha rhythm results in enhanced power and phase-locking, and, in turn, the entrained alpha power and phase modulate perception, consistent with findings on the effects of spontaneous oscillations (Romei et al., 2010; Spaak et al., 2014; Thut et al., 2011).

In short, there is both correlational and causal evidence that ongoing alpha oscillations shape visual perception.

\subsection{Alpha activity plays an inhibitory role}

The direction of behavioural effects of alpha activity described in the literature, i.e., a larger alpha power results in a lower the probability of perceiving a visual stimulus, has led to the most widespread interpretation that alpha activity plays an inhibitory role (Klimesch et al., 2007Jensen and Mazaheri, 2010; but see Palva and Palva, 2007).

According to the gating by inhibition hypothesis (Jensen and Mazaheri, 2010), alpha inhibition appears to be cyclic and linked to the phase of alpha oscillations: rhythmic alpha activity serves as a pulsed inhibition that modulates the time window for sensory processing, i.e., the duty cycle. In this view, when alpha power is high (i.e., high inhibition), the duty cycle is shorter than that when alpha power is low (Jensen and Mazaheri, 2010; Mathewson et al., 2009), as shown by the green trace in Fig. 2A.

Furthermore, alpha oscillations may be "asymmetric" or "biased" (Hyafil et al., 2015; Jensen and Mazaheri, 2010; Schalk, 2015), meaning that alpha oscillations are not zero-mean. Rather, the mean of an alpha cycle varies with the amplitude of alpha oscillation, as shown by the red trace in Fig. 2A. According to this perspective, the instantaneous voltage level may represent a more direct measure of functional inhibition than power and phase alone, as suggested by the function-through-biased-oscillations (FBO) framework (Schalk, 2015;
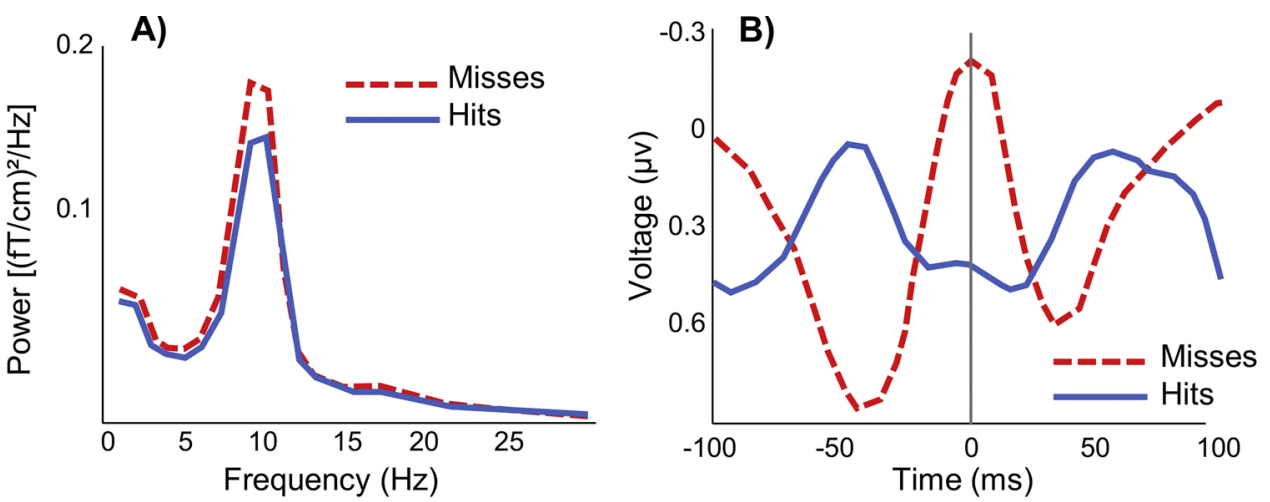

Fig. 1. Effects of prestimulus M/EEG alpha oscillations on visual detection. A) Lower alpha power preceding the stimulus leads to a higher proportion of hits (grand average of the spectra calculated in the second before target onset in occipital MEG channels; adapted from van Dijk et al., 2008). B) Opposite alpha phase at target onset (time: $0 \mathrm{~ms}$ ) for hits and misses (grand average event-related potential at EEG channel Pz; adapted from Mathewson et al., 2009). 


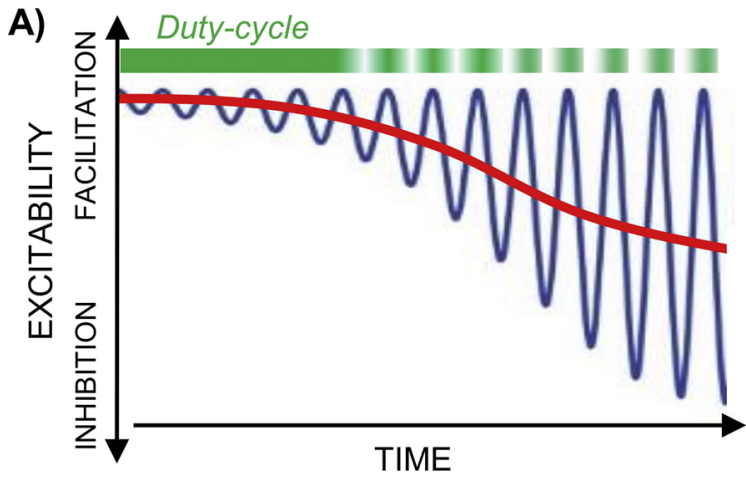

Schalk et al., 2017). A higher amplitude of alpha activity (in absolute value) corresponds to a more negative instantaneous voltage level, which leads to higher inhibition (Hyafil et al., 2015; Fig. 2B). Such interpretation is supported by invasive neurophysiological recordings, showing both a general decrease in neural firing rate during periods of high alpha power and a rhythmic relation between alpha oscillations and neuronal spiking (Haegens et al., 2011). FBO predictions are consistent with evidence showing the effects of alpha phase only when alpha power is high (Cohen and Van Gaal, 2013; Mathewson et al., 2009).

Concisely, negative asymmetric alpha oscillations represent a key element in inhibition.

\subsection{Alpha inhibition through alpha-gamma cross-frequency interactions}

Existing evidence (Bahramisharif et al., 2013; Jensen et al., 2014; Roux et al., 2013; Spaak et al., 2012) and current theories on the functional role of the alpha band (Bonnefond et al., 2017; Jensen and Mazaheri, 2010) suggest that the pulsed inhibition caused by alpha oscillations, which shortens the duty cycle, occurs by means of crossfrequency interactions with the gamma band. Accordingly, alphagamma interactions have been consistently observed during rest and in the prestimulus window using both invasive and non-invasive M/EEG recordings (Bahramisharif et al., 2013; Osipova et al., 2008; Spaak et al., 2012; but see Ray and Maunsell, 2015). According to these results, in addition to the neural gamma activity associated with stimulus processing (Fries et al., 2007), alpha-gamma coupling is likely integrated in the ongoing activity that shapes the perceptual outcome (Ni et al., 2016; van Es and Schoffelen, 2019v).

Hereafter, we will focus on the coupling between the alpha and gamma band, while an in-depth analysis of gamma band activity in stimulus coding and its role in perception is provided in the study by Pritchett et al. (2015).

As shown in Fig. 3, the relationship between alpha and gamma oscillations is regulated both by amplitude-amplitude coupling (AAC), i.e., alpha power increase associated with gamma decrease, and by phase-amplitude coupling (PAC) interactions, i.e., gamma power nested within the alpha phase (Spaak et al., 2012). In line with the asymmetry in alpha oscillations, the stronger the alpha amplitude, the stronger the PAC (Osipova et al., 2008); high-amplitude alpha oscillations, accompanied by a general decrease in alpha voltage level, can lead to a stronger phasic modulation of gamma power compared to low-amplitude alpha oscillations (Hyafil et al., 2015). Therefore, PAC describes the phasic suppression of macro-scale M/EEG gamma activity within an alpha cycle.

In summary, we can conclude that alpha-gamma cross-frequency interactions are involved in ongoing oscillatory activity, in which alpha oscillations inhibit M/EEG gamma power.

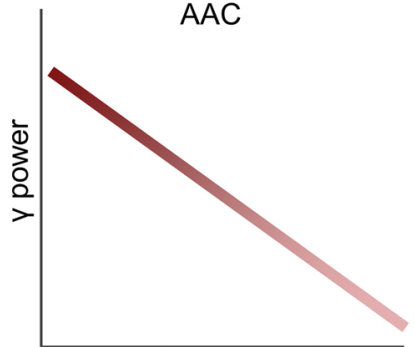

$\alpha$ amplitude
Fig. 2. Negative and asymmetric alpha oscillations. A) An increase in alpha amplitude is accompanied by a modification in the mean voltage level (blue trace: time-varying instantaneous voltage; red trace: mean voltage level), which shortens the duty cycle (green trace); adapted from Schalk (2015). B) Alphaband oscillations (blue trace) modulate neuronal spiking activity (bottom); neural spiking is phasically reduced, i.e., pulsed inhibition is stronger when the amplitude of alpha oscillations is higher; adapted from Hyafil et al. (2015).
Fig. 3. Schematic representation of alpha $(\alpha)$-gamma $(\gamma)$ cross-frequency interactions. Left: Amplitude-amplitude coupling (AAC); gamma power decreases as a function of alpha amplitude. Right: Phase-amplitude coupling (PAC) at different alpha amplitudes; when alpha is low (dark colour), the averaged gamma power (dashed line) is high with negligible influence of alpha phase, while when alpha is high (light colour), the averaged gamma power is low on average (dashed line) and is nested within the alpha cycle. Note that the maximum value of gamma power is the same for low and high alpha inhibition. Adapted from Hyafil et al., 2015.

\subsection{Alpha-modulated gamma oscillations affect the probability of response to incoming stimuli}

The alpha-gamma AAC and PAC mechanisms are grounds to understand the inhibitory effects of ongoing alpha oscillations on perception: alpha can modulate the probability of response of gamma-oscillating neurons of sensory areas to incoming stimuli of different intensities (i.e., luminance or contrast level, considered to be proportional to the input to neurons and quantified in terms of depolarizing current; Tiesinga et al., 2004) and, consequently, the behavioural response probability.

Before taking into account alpha modulation of gamma activity, let us consider more deeply the probability of response of gamma oscillations. Gamma oscillations of small groups of neurons can be measured at the meso-scale level by means of invasive recordings, such as LFP, and more specifically, its local component (Kajikawa and Schroeder, 2011). Fig. 4A shows the probability of response, according to the OPR model, when facing a wide range of input intensities in a condition without alpha inhibition: the neural response probability depends on a threshold (to be reached to induce a response), input intensity and amplitude of LFP gamma oscillations (Ni et al., 2016). If we consider a wide range of input intensities below a critical intensity (a), an incoming input will never induce a neural response (response probability $=0$ ) in sensory areas. For higher intensities, the neural response depends on the phase and amplitude of LFP gamma oscillations, giving rise to a response probability between 0 and 1 . For even higher intensities, i.e., above a value (b), the stimulus will induce a response independently of gamma phase (response probability $=1$ ). The resulting probability function of the neural response (from now on referred to as "single" probability of response) at different input 


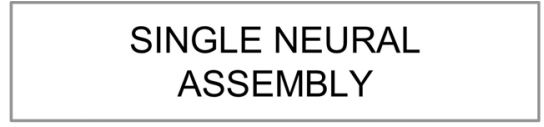

A)

Low-intensity input

High-intensity input

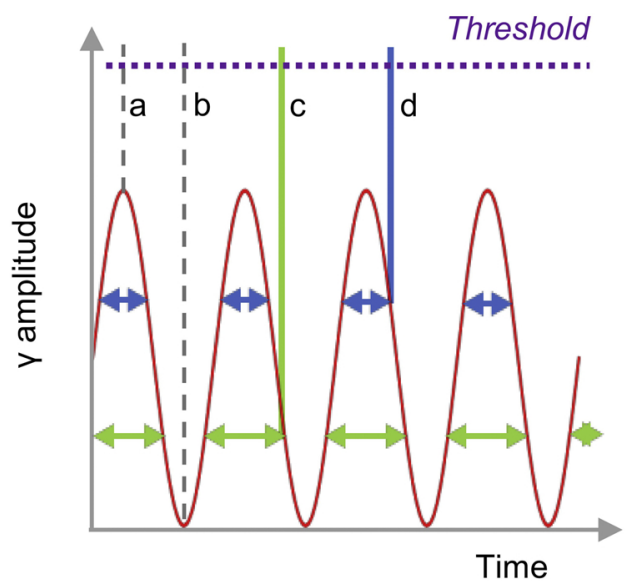

B)

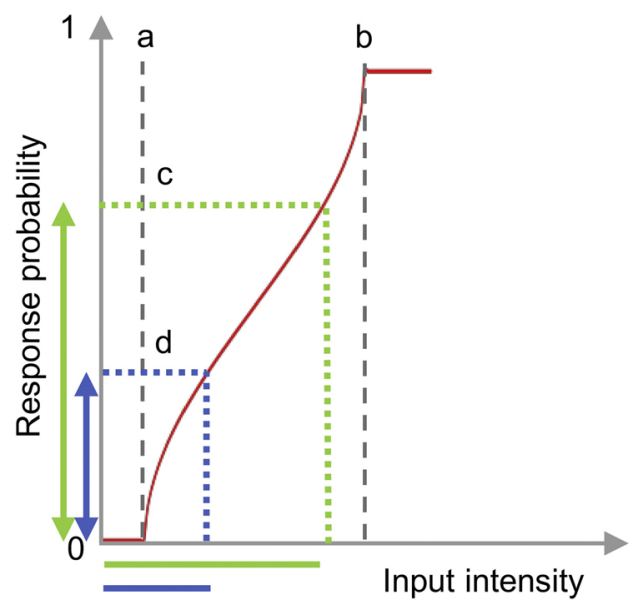

NEURAL POPULATION

C)

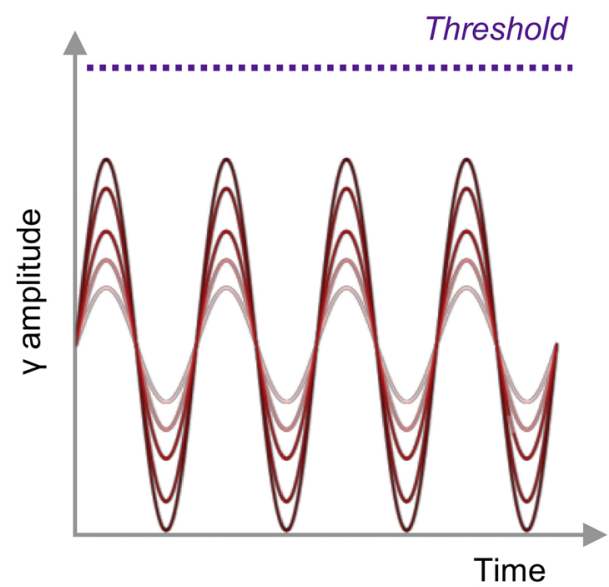

D)

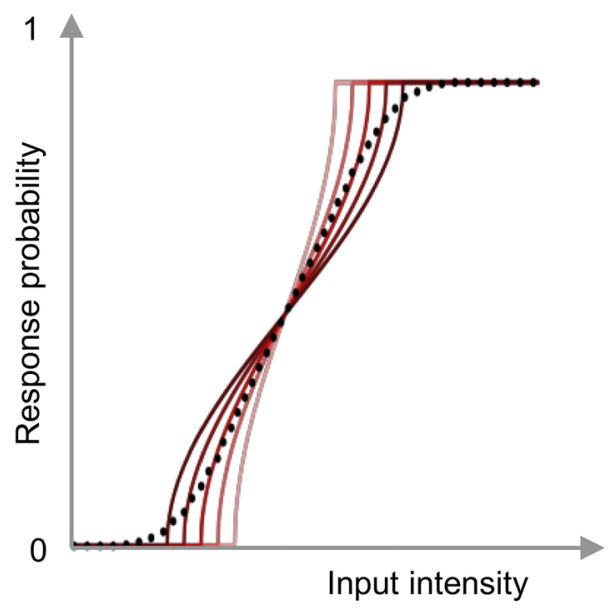

Fig. 4. Neural response probability as a function of input intensity in a condition without alpha inhibition. A) Incoming inputs (c: high intensity, green; $d$ : low intensity, blue) during LFP gamma oscillations of a small group of neurons in sensory areas. Given a fixed threshold for response, double-headed arrows indicate the response probability (i.e., time windows during which incoming inputs may induce a response). B) Single probability of response: response probability as a function of input intensity at fixed threshold and fixed gamma amplitude, as defined in (3). Along the input intensity axis, $a$ represents the minimum input intensity able to elicit neural response, $b$ shows the minimum input intensity that determines a response independently of phase, and inputs between $a$ and $b$ indicate intensities inducing a phase-dependent response (probability between 0 and 1); $c$ and $d$ represent the high- and low-intensity inputs shown in (A). Along the response probability axis, $c$ and $d$ represent the response probability of the highand the low-intensity inputs, respectively. C) Variability in the amplitude of LFP gamma oscillations within the neural population leads to (D) variability in response probability functions. The mean of all response probability functions, weighted for their distribution in the neural population, results in a sigmoid function that represents the global response probability function (dotted line). intensities is represented in Fig. 4B and mathematically defined as follows (formulas reported in Table 1). LFP gamma oscillations $\left(G_{F}\right)$ are defined in (1) as a sinusoidal function, while the single probability of response $\left(P_{S R}\right)$, independent of gamma frequency, is defined in (2), which is calculated as the ratio between the time window in which the input elicits a response (i.e., when the sum of gamma voltage and input intensity is higher than the threshold) and the wavelength of gamma. Importantly, this calculation is based on gamma features at the immediate time of stimulus presentation and does not require the stationarity assumption, i.e., the signal is not required to be stable for one or more gamma cycles. The sinusoidal function of gamma cycles has the only aim to model the random nature at which a stimulus can occur and with the same probability in each phase of a gamma cycle, but it does not indicate that the signal must to be stable at the time when the stimulus is presented. The only requirements are that the stimulus occurs randomly in the gamma cycle and that a sufficient number of stimuli are presented for an accurate representation of the probability. When considering a population of neurons (i.e., many small groups of neurons), the global probability of response is calculated by averaging the single response probabilities. Within a neural population, we can assume a certain degree of variability in the amplitude of LFP gamma oscillations (Fig. 4C). Considering this variability to be normally distributed among neurons, the global response probability can be expressed as the weighted average of single response probability functions, as defined in (3). As shown in Fig. 4D, the global response probability reveals a sigmoid trend.

Crucially, the OPR allows us to estimate the probability of response when gamma activity is modulated by pulsed alpha inhibition by considering several features of the alpha-gamma interaction. First, the gamma band oscillates around the alpha voltage level, as is typically detected in raw LFP signals (Jia and Kohn, 2011). Therefore, because alpha oscillations are asymmetric, gamma activity is shifted closer to or further from the threshold in a phase-dependent manner locked to alpha oscillations. Moreover, gamma activity is regulated by alpha activity through mechanisms that generate the AAC and PAC observed in M/EEG: an increase in alpha power leads to a stronger modulation of gamma activity with a stronger phase dependency. The link between the responses of neural populations to the behavioural outcome is a highly complex subject, but we can assume that the behavioural response probability is proportional to the response at the neural level, at least in simple visual perception tasks such as detection (Britten et al., 1992; Reynolds et al., 2000; Williford and Maunsell, 2019; but see Hara et al., 2014). Therefore, we consider that the global response probability function obtained from (3) may represent the psychometric 


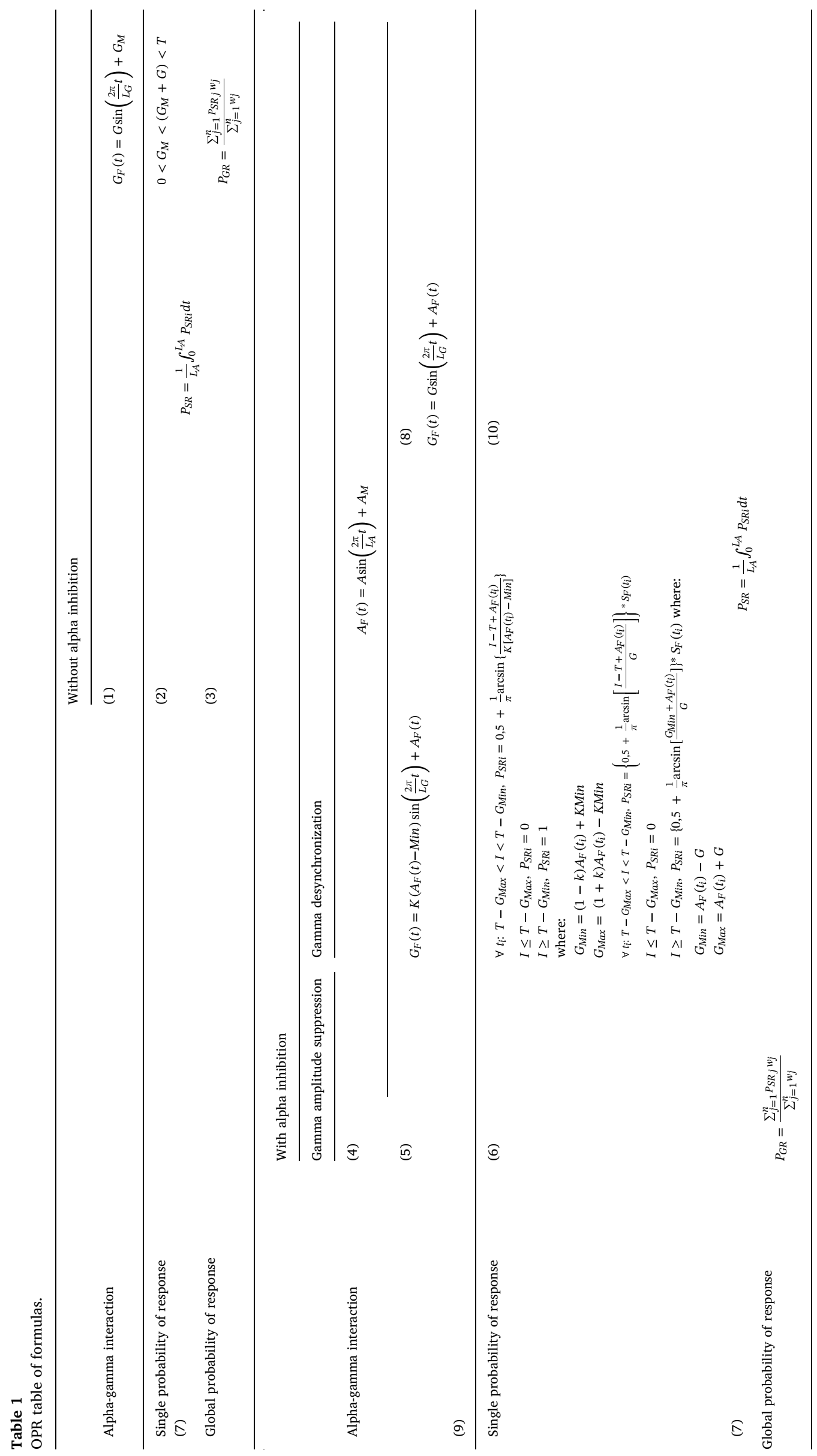


function observed behaviourally (Britten et al., 1992), in which an observer's performance in a visual detection task is related to the physical quantity of a stimulus, e.g., its intensity (Wichmann and Hill, 2001).

As explained above, the calculation of the probability of response of gamma oscillating neurons does not require the stationarity assumption. Thus, the model is suitable to represent the modulation of gamma activity by alpha oscillations. Finally, alpha oscillations themselves do not need to be stationary, although a sinusoidal function is used to represent the randomness of the occurrence of the stimulus in the alpha cycle.

The OPR models two different meso-scale mechanisms that regulate alpha-gamma interaction. Notably, these two meso-scale mechanisms would result in the same alpha-gamma modulation at the macro-level of M/EEG oscillations, but lead to distinct effects on the final behavioural response, as we will describe in detail in the next section.

In sum, these observations reveal that the OPR predicts the probability of response of gamma-oscillating neurons of sensory areas and therefore the probability of behavioural response, which is mediated by alpha activity.

\subsection{The probability of response to incoming stimuli is specifically modulated by distinct coupling mechanisms of alpha-gamma interactions}

Despite compelling evidence about the inhibitory role of alpha activity on visual perception and on cross-frequency interactions with the gamma band, little is known about the micro- and meso-scale mechanisms associated with ongoing alpha inhibition (Cohen, 2017; Hyafil et al., 2015; Sadaghiani and Kleinschmidt, 2016; Spaak et al., 2012). Indeed, the general relation between $M / E E G$ features and lowerscale mechanisms is likely to be few to some rather than one to one: the same M/EEG oscillatory feature (e.g., alpha activity) may be generated by distinct processes (Cohen, 2017). For example, at lower-scale levels, M/EEG power appears to be generated both by changes in amplitude, i.e., the magnitude of an oscillation (Whittingstall and Logothetis, 2009) and in synchronization, i.e., the temporal alignment of the phase of oscillations (Makeig et al., 2002). Specifically, evidence from simultaneous LFP and EEG recordings of the visual cortex in behaving monkeys has shown that LFP amplitude and synchronization independently contribute to M/EEG gamma power (Musall et al., 2014). Therefore, distinct modulations of LFP gamma oscillations at the mesoscale potentially result in the same effects on the macro-scale M/EEG gamma oscillations.

Based on this evidence, the OPR models these two mechanisms in the alpha-gamma cross-frequency interaction to explore their effects on the probability of response to incoming visual inputs. Importantly, according to the OPR, alpha-driven changes in amplitude and in synchronization of LFP gamma oscillations lead to distinct effects on the global response probability function and therefore on the behavioural outcome. The OPR independently models the effects of changes in the amplitude and synchronization of the gamma band. Although we are unable to exclude the possibility that the two mechanisms co-occur simultaneously, the OPR may still be able to disentangle these mechanisms if one prevails over the other.

Thus, a psychophysical approach that describes behavioural responses for a wide range of stimuli, i.e., the psychometric function, provides a powerful tool that may be exploited to disentangle the effects of the two phenomena in alpha-driven modulation of sensory processing.

According to the OPR, alpha modulates M/EEG gamma power by decreasing (a) the amplitude of LFP gamma oscillations and/or (b) the degree of synchronization among small groups of neurons.

(a) Alpha reduces LFP gamma amplitude. An increase in alpha oscillations may induce not only a rhythmic decrease in the mean LFP gamma voltage level but also a decrease in gamma amplitude, which leads to a reduction in M/EEG gamma power (Fig. 5, blue box).
Similarly, the amplitude of fast oscillations has been shown to be increased by depolarizing currents that bring membrane potentials closer to the firing threshold (Bracci et al., 2003). With the same mechanism, alpha activity may phasically move the mean voltage of gamma oscillations away from threshold inducing a phasic reduction of these oscillations, the mechanism of which is mathematically defined as follows. Alpha $\left(A_{F}\right)$ is defined in (4), and alpha-modulated gamma $\left(G_{F}\right)$ is defined in (5): LFP gamma oscillation is modelled as a sinusoidal function with a mean equal to alpha voltage and amplitude proportional to alpha voltage. In this way, the gamma phase is independent of the alpha phase, so that at each time point in the alpha wave, the gamma phase can assume any value. In (6), the probability of response of neurons $\left(P_{S R}\right)$ is defined for each time point $\left(t_{i}\right)$ in combination with (2), which calculates the single response probability for gamma oscillations, and with (4) and (5), which include the modulation of gamma oscillations by alpha activity. The single probability of response of neurons over the entire alpha cycle for each input intensity is calculated as the sum of response probabilities at each time point $t_{i}$ with time interval $d t$, with $d t$ close to zero, the calculation becomes more precise and is expressed by (7). As defined above, when considering a population of neurons, the global response probability $\left(P_{G R}\right)$ is expressed as the weighted average of single response probabilities (3) and results in a sigmoid trend.

A change in alpha activity modulates the psychometric function and determines a horizontal shift that can be calculated with a numerical simulation expressed by (6), (7) and (3). When alpha increases, the psychometric function is shifted rightwards, resulting in a worsening of visual performance, as shown by the blue trace in Fig. 5 .

In the literature on visual perception, a shift of the function is commonly described in terms of a contrast gain mechanism, arising from a divisive scaling of the input (Chaumon and Busch, 2014; Ling and Carrasco, 2006; Pestilli et al., 2007; Reynolds et al., 2000; van Boxtel, 2017). Interestingly, a contrast gain-like mechanism has been more frequently associated with the activity of single neurons than the activity of the entire system (Kim et al., 2007), consistent with the OPR. Moreover, a contrast gain-like mechanism has been suggested as the main functional process underlying sustained endogenous attention and adaptation, both from behavioural studies and single-unit neural measurements (Cameron et al., 2002; Donovan and Carrasco, 2018; Pestilli et al., 2007; Reynolds et al., 2000). However, the relationship between alpha-gamma oscillations, attention and perceptual outcome assessed using the psychometric function has not yet been investigated.

(b) Alpha reduces LFP gamma synchronization. Alpha-driven gamma power modulations may otherwise arise from phasic desynchronization of LFP gamma oscillations (Fig. 5, violet box). In this case, the alphainduced rhythmic decrease in the gamma mean is associated with a decrease in synchrony. The degree of neural synchronization is expressed as values ranging from 0 to 1 , where 0 represents complete gamma desynchronization among neurons and consequent zeroing of the global response, despite the actual response of single neurons. This definition is in line with several measures adopted to quantify neural synchrony in the literature on functional connectivity, which rely on the concept of cross-correlation (e.g., the coherence coefficient; Bastos and Schoffelen, 2016). Here, alpha $\left(A_{F}\right)$ is defined in (4), alpha-modulated gamma $\left(G_{F}\right)$ is defined in (8), and synchronization is modelled in (9), as follows. The synchronization factor $\left(S_{F}\right)$, as expressed in (9) and shown in Fig. 6, is modelled to be proportional to the alpha oscillation, varying in a range that is limited by the maximal and minimal voltages that alpha activity is able to reach, as defined in (4). The synchronization factor is 1 when the alpha voltage is at its maximum value, the synchronization factor is 0 when the alpha voltage is at the absolute minimum voltage, and the value of the synchronization factor at each time point of the alpha wave is proportional to the difference between the immediate alpha voltage and absolute minimum voltage. Therefore, the synchronization factor depends on the immediate voltage of alpha in relation to an absolute minimum rather than the minimum of the 


\section{ALPHA INHIBITION}

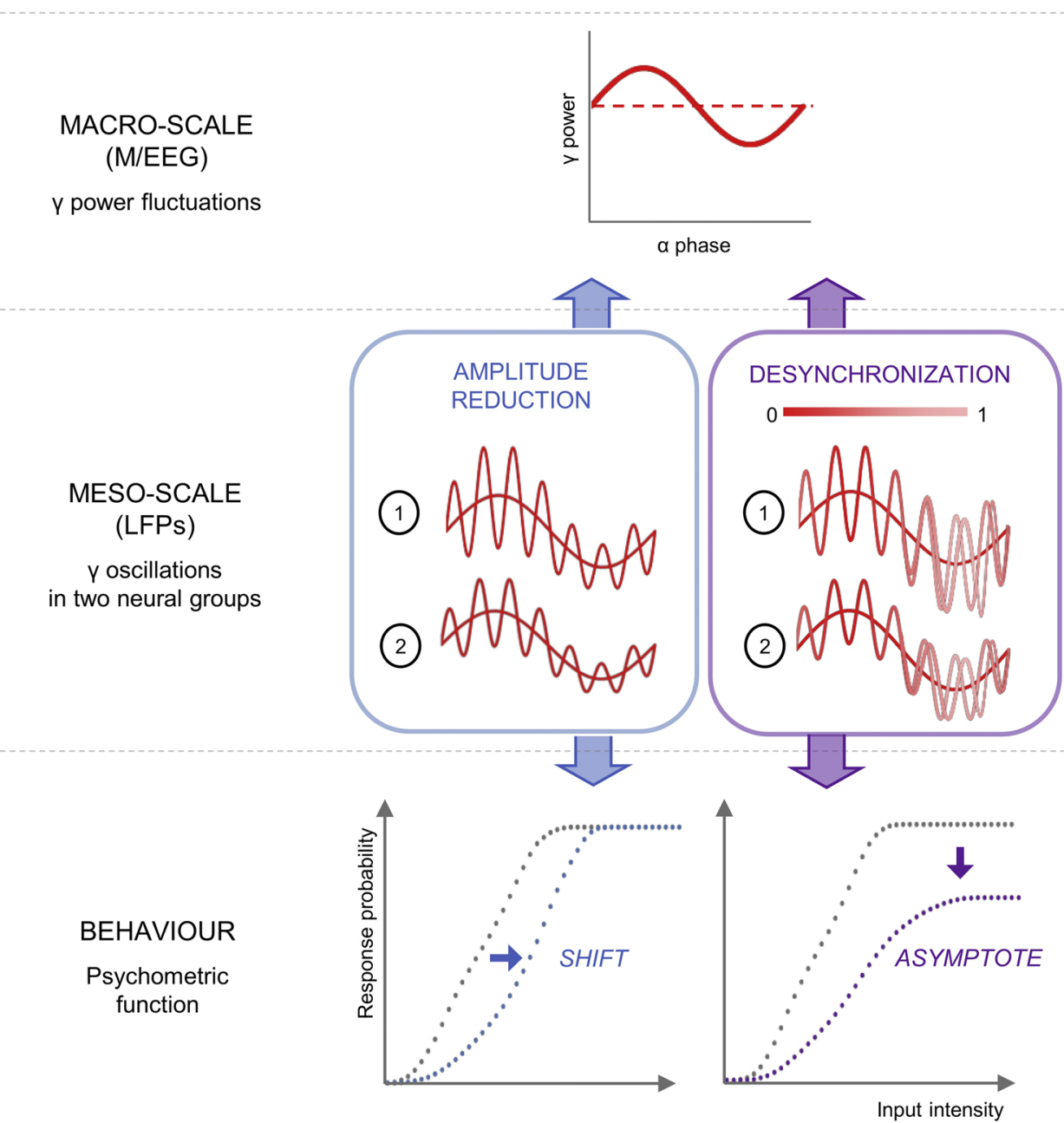

Fig. 5. The OPR predictions associated with alpha inhibition described at different scales. Macro-scale (top row): Gamma power, as measured by M/EEG recordings, is modulated by alpha inhibition by means of AAC and PAC mechanisms shown in Fig. 3: M/EEG gamma power is lowered on average and is nested within the alpha phase. Meso-scale (middle row): Two representative groups of neurons in primary sensory areas, oscillating at gamma frequency with different amplitudes, as measured by the LFP, that are modulated by alpha activity. During alpha inhibition, the modulation of gamma power fluctuations may arise either from a phasic suppression of gamma amplitude (blue box) or from a phasic desynchronization of gamma oscillations (violet box; synchronization range as shown by colour bar). Behaviour (bottom row): two mechanisms of alpha inhibition lead to distinct effects on the psychometric function (dashed line: psychometric function without alpha inhibition). If alpha inhibition is associated with a phasic suppression of the amplitude of gamma oscillations, the OPR predicts a rightward shift of sensory threshold (blue line), while phasic gamma desynchronization is expected to give rise to a lowering of the upper asymptote (violet line). alpha cycle in which gamma is embedded and does not depend on the stationarity of the signal.

Postulating that the LFP gamma phase is independent from the alpha phase, the single probability of response is defined for each time point $\left(t_{i}\right)$ in (10), by combining (2) with (4) and (8), multiplied by the synchronization factor (9). Then, the single $\left(P_{S R}\right)$ and the global $\left(P_{G R}\right)$ probabilities of response are calculated in (7) and (3), respectively, consistent with that described in the previous paragraph. A numerical simulation of the psychometric function expressed by (7) and (10) results in a sigmoid trend. As shown by the violet trace in Fig. 5, an increase in alpha activity associated with phasic gamma desynchronization leads to a worsening of visual performance, in this case by lowering the upper asymptote.

In the literature on visual perception, a modification in the upper

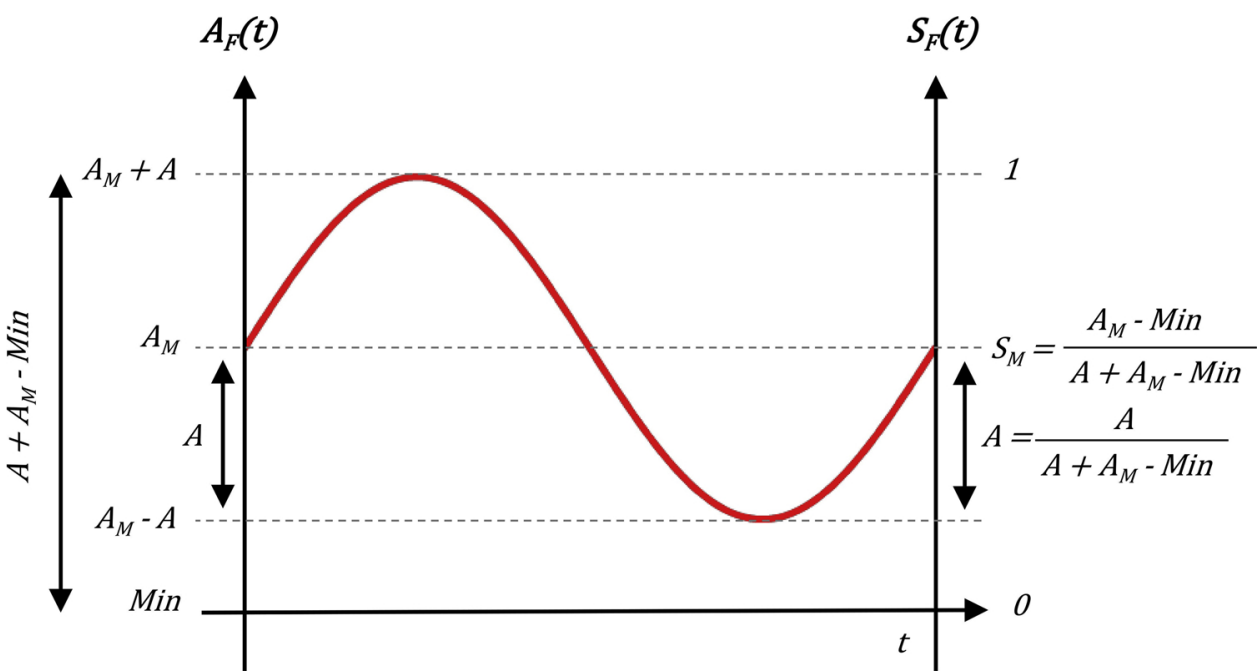

Fig. 6. Synchronization factor. The synchronization factor $\left(S_{F}(t)\right)$ is defined as a sinusoidal function synchronized with $A_{F} . S_{F}(t)$ is 1 when $A_{F}(t)$ is maximal (i.e., $A_{F}(t)=A_{M}+A$ ); 0 on the $\mathrm{S}_{\mathrm{F}}$ axis corresponds to the minimum voltage (Min) on the $A_{F}$ axis, where Min is the absolute minimum voltage that $A_{F}$ has the potential to reach. Therefore, interval 1 on the $S_{F}$ axis corresponds to interval $A_{M}+A-M i n$ on the $A_{F}$ axis, making the following proportions valid: $\mathrm{S}_{\mathrm{M}}: \mathrm{A}_{\mathrm{M}}=1:\left(\mathrm{A}+\mathrm{A}_{\mathrm{M}^{-}}\right.$Min); $\mathrm{S}: \mathrm{A}=1:(\mathrm{A}$ $+\mathrm{A}_{\mathrm{M}^{-}}$Min). 
bound of the psychometric function is referred to as the response gain mechanism, which mainly affects performance (as well as the neural response) to high-intensity stimuli because the modulation is proportional to the response (Chaumon and Busch, 2014; Reynolds et al., 2000). Intriguingly, neural synchronization has been proposed as a candidate mechanism for response gain (Buia and Tiesinga, 2006; Fries et al., 2001; Kim et al., 2007; Reynolds and Chelazzi, 2004). In previous studies investigating the relationship between attention and visual perception, the response gain mechanism has been related to transient and exogenous attention (Ling and Carrasco, 2006; Pestilli et al., 2009; Wang and Krauzlis, 2018), although a direct link with ongoing oscillations has not been reported.

In summary, the OPR predicts distinct effects of an alpha-induced LFP gamma amplitude decrease and gamma desynchronization on the psychometric function, i.e., a rightward shift of the curve and a lowering of the upper asymptote, respectively.

Here, alpha is represented as a stationary function for simplicity. However, the calculation of the synchronization factor is based on the immediate alpha voltage and does not depend on the stationarity assumption within one alpha cycle.

\section{Discussion and future directions}

The OPR models the behavioural outcomes of distinct meso-scale mechanisms that may be associated with the same M/EEG feature, i.e., ongoing alpha oscillations and cross-frequency alpha-gamma interactions. Our framework is based on consistent evidence from non-invasive electrophysiology revealing the effects of ongoing M/EEG alpha oscillations on visual perception, and it aims to explore the link with other fields of investigation, such as the psychophysical approach to visual perception and invasive recordings.

The effects of ongoing alpha oscillations reported in the literature are consistent. Nevertheless, these effects may represent an underestimation of the actual relationship between oscillations and perceptual outcome. The computation of the oscillatory activity during the "prestimulus window" to estimate its effects at the time of stimulus presentation is based on the assumption that the signal is stationary in the analysed window and enables predictions of the exact moment along the oscillation cycle in which an external input reaches the cortex. However, according to recent studies, oscillatory activity may not be sustained but consists of burst-like events (van Ede et al., 2018v). Thus, the effects of ongoing oscillations may be better represented by the status of the cortex at the time of stimulation, consistent with the findings reported by Romei and coworkers (Romei et al., 2012). The OPR does not rely on the stationarity assumption, as explained in the previous paragraphs; its predictions are based on the exact point in time of the incoming stimulus and can thus be extended to peristimulus burst-like activity. The necessary condition on which the OPR relies to provide an accurate estimation of the probability of response is that a sufficient number of stimuli are presented randomly along the alpha cycle.

The predictions of the OPR strictly rely on alpha-gamma coupling and they would not be valid if only alpha or only gamma oscillations were considered. On one hand, the OPR relies on gamma, because it defines the probability of response of gamma-oscillating neurons, while alpha is modelled as the oscillatory activity of neurons that are not directly involved in stimulus processing. When considering alpha alone, concepts such as changes in excitability and changes in duty cycle that are incorporated in the OPR might still be formulated, and they have indeed been proposed in other models before the OPR. However, distinct effects of alpha on the psychometric function would not be dissociable. In fact, the OPR provides predictions for the effects of alpha by exploiting a mathematical model of distinct types of meso-scale alphagamma coupling.

On the other hand, the OPR relies on alpha. Indeed, the OPR aims to explain the effects of alpha, and the predictions of the OPR strictly depend on the modulatory effects of low-frequency oscillation, i.e., in the alpha band, on high-frequency oscillations, i.e., in the gamma band. When considering gamma alone, the model would lack two fundamental mechanisms: a) changes in amplitude/synchronization of gamma oscillations occur phasically along the alpha cycles and b) the average gamma level phasically shifts further from or closer to the threshold with the alpha cycle. Therefore, while mathematical predictions of how changes in gamma amplitude/synchronization shape perception are still possible based on gamma oscillations alone, they would not explain the alpha inhibitory mechanisms.

Clearly, the OPR is intended as a simplification of the relationship between neural mechanisms and behaviour, and future studies combining simultaneous multiscale recordings are needed to validate the OPR. First, concurrent LFP and EEG in animal models will be critical in establishing whether LFP gamma synchronization and oscillations amplitude are associated with ongoing EEG alpha activity and whether the two mechanisms can dissociate. Moreover, to the best of our knowledge it is still unclear which neurophysiological factor could determine the occurrence of one coupling mechanism or the other one. Future studies may elucidate whether these mechanisms are due to distinct alpha generators, which may be responsible of different signal characteristics (e.g., peak frequency or burstiness), and/or to different properties of neurons in primary sensory areas, which may respond differently to the same alpha oscillations. Finally, the specific effects of these meso-scale mechanisms associated with alpha oscillations (i.e., LFP gamma amplitude decrease and desynchronization) on neural responses and perceptual outcome may be explored during a visual detection task involving different stimulus intensities. Importantly, we emphasize that the two distinct effects on the psychometric function (i.e., a shift $v s$. change in the upper asymptote) must be tested, as reported in the studies by (Chaumon and Busch, 2014; Ling and Carrasco, 2006; Reynolds and Chelazzi, 2004).

\subsection{OPR applications}

At the present stage, valuable insights into the context of non-invasive recordings may arise from the general approach that the OPR suggests, i.e., investigating the effects of M/EEG ongoing oscillations through the psychometric function. Specifically, the OPR may provide insights into the open question of whether the ongoing M/EEG alpha rhythm represents a non-unitary phenomenon by revealing the existence of different processes associated with the same macro-scale phenomenon (i.e., alpha oscillations) and formulating hypotheses about the involvement of meso-scale mechanisms.

Fields of application of the OPR are numerous and may include perceptual and attentional processes.

In the context of perception, the OPR allows to interpret the spontaneous variability of visual detection, which has been associated with alpha fluctuations. In this field, only one study investigated the effects of prestimulus alpha activity on visual performance by relating the ongoing alpha power to changes in the psychometric curve as a function of stimulus intensity (Chaumon and Busch, 2014). Chaumon and Busch (2014) observed an association between a higher prestimulus alpha power and a decrease in the upper asymptote of the psychometric function (and not a leftward shift), which has been interpreted as reflecting a response gain mechanism. The OPR allows us to interpret the specific modifications of the psychometric function observed in this visual task based on the underlying neural mechanisms, in this case, a desynchronization of gamma activity in visual areas. Future studies involving invasive recordings and employing the same perceptual paradigm may confirm this hypothesis.

Another field of application is the relation between ongoing alphagamma oscillations, visual attention and perceptual outcome. In the M/ EEG literature on ongoing alpha activity, the same oscillatory process has been suggested to subtend attentional mechanisms, regardless of the type of attention (i.e., spatial, temporal, selective, and internal 
attention; for a review see Frey et al., 2015). However, researchers have not clearly determined whether the same process operates in all these cases. We therefore suggest that a psychophysical approach, which has proven to be extremely valuable in the literature of visual perception (Cameron et al., 2002; Herrmann et al., 2010; Pestilli et al., 2009, 2007; Pham and Kiorpes, 2019; Reynolds et al., 2000; van Boxtel, 2017; Wunderle et al., 2015), may allow to disentangle possible mechanisms of alpha inhibition associated with distinct types of attention. For example, different types of visual attention lead to distinct behavioural effects, as assessed using the psychometric function (Donovan and Carrasco, 2018; Ling and Carrasco, 2006). Sustained/endogenous attention affects the perceptual threshold (contrast gain), while transient/ exogenous attention also affects the upper asymptote of the psychometric function (response gain). Based on this evidence, we expect that future M/EEG studies will find that (1) ongoing alpha activity is related to both endogenous and exogenous attention and (2) the two processes differ in their effects on the psychometric function (i.e., a shift of the function and modification of the upper asymptote, respectively). If these hypotheses are confirmed, the OPR would then predict that endogenous and exogenous attention are associated with different mesoscale mechanisms in the gamma band, i.e., the oscillatory amplitude and synchronization modulation, respectively.

Finally, the OPR is also a potentially useful tool to investigate the effects of alpha activity and attention on processes throughout the visual hierarchy. The effects of spatial attention on neural receptive fields have been shown to be comparable throughout the visual stream in humans (Klein et al., 2014), although researchers have not determined whether the effects of alpha oscillations are the same. If these effects were confirmed, a reasonable hypothesis would be that alpha-induced gamma desynchronization prevails in downstream stages along the visual pathway, which require the integration of several visual features (Buzsáki and Draguhn, 2004). Conversely, an alpha-induced decrease in the gamma amplitude might be observed in the very first stages of the visual hierarchy, maximizing the sensitivity of small groups of neurons that are selective for basic stimulus features. Thus, stimulus complexity may be a relevant factor to consider in the application of OPR, consistent with the normalization model of attention (Reynolds and Heeger, 2009), which suggests that the attentional effects of contrast gain and response gain on the neurometric function also depend on stimulus properties. A crucial step for future studies will be to integrate this thriving field of study of the attentional effects on neural coding with research on ongoing neural oscillations.

\subsection{Generalization of the $O P R$ to other frequencies and to other perceptual} domains

The OPR models the modulation of high-frequency oscillations involved in stimulus processing by an inhibitory slow-frequency rhythm and determines its effects on perception. While the OPR arises from the literature on visual perception and alpha-gamma oscillations, from both a mathematical and physiological perspective, it models a general mechanism and therefore could be extended to the investigation of other frequency bands and other perceptual domains.

For example, visual perception has also been shown to be phasically modulated by slower frequencies in the theta range $(4-7 \mathrm{~Hz})$ (Fiebelkorn et al., 2018; Landau and Fries, 2012), and alpha and theta rhythms behave similarly in terms of the cross-frequency coupling with the gamma band (Helfrich et al., 2018; Landau et al., 2015). On the other hand, the two frequency bands can dissociate. An intriguing hypothesis suggests the existence of a faster "occipital alpha" at $\sim 11 \mathrm{~Hz}$, which is involved in sensory aspects of perception to a greater extent, and a slower "frontal alpha/theta" at $\sim 7 \mathrm{~Hz}$, which appears to be implicated in visual attention (Sherman et al., 2016; Zoefel and VanRullen, 2017). This hypothesis suggests different generators for the two rhythms, and it is supported by a recent MEG study performed during the resting state, in which source-level analyses showed alpha (but not theta) oscillations in occipital regions and theta (but not alpha) activity in the inferior frontal gyrus (Keitel and Gross, 2016). Consistent with these findings, Harris and colleagues have shown that while the alpha rhythm predicts visual performance both for attended and unattended stimuli, the theta band accounts for the detection of unattended stimuli alone (Harris et al., 2018). In this context, the OPR may also be useful in exploring whether alpha and theta rhythms subtend different processes to explain the effects of ongoing oscillations on perceptual outcome. In this regard, a non-trivial issue to consider in future research is to control for methodological limitations, such as an a priori focus on a pre-defined frequency band in the analysis of brain oscillations.

Furthermore, similar effects to the ones described for ongoing oscillations on visual perception have been described in the somatosensory (Baumgarten et al., 2016; Haegens et al., 2011; LinkenkaerHansen, 2004) and auditory domains (VanRullen et al., 2014; Zoefel and VanRullen, 2017), suggesting that alpha activity may subtend a general physiological mechanisms (Haegens et al., 2015). Consistent with this evidence, the OPR may be generalized not only to other frequency bands, but also to other perceptual domains. However, investigations of the relationship between oscillations and perception beyond near-threshold stimuli in these domains remain scarce, and further studies are needed to understand the possible oscillatory mechanisms underlying perceptual behavioural outcome.

\section{3. $O P R$ and current theories}

As extensively outlined in the manuscript, the model is based on fundamental theories of neural oscillations and alpha inhibition (Bonnefond et al., 2017; Jensen and Mazaheri, 2010; Klimesch et al., 2007; Schalk, 2015; VanRullen and Dubois, 2011). Here, we briefly discuss how the OPR fits into other perspectives of the role of alpha activity in perception.

The OPR shares a few similarities with the framework proposed by Jensen and colleagues (Jensen et al., 2012). Indeed, both models address the relationship between alpha-gamma cross-frequency coupling and visual perceptual outcome, proposing possible candidates for the underlying neural mechanism. Jensen et al. (2012) describe a phase code inspired by the theta-gamma mechanism observed in the rat hippocampus, through which alpha activity prioritizes unattended visual inputs and orders them according to relevance. Specifically, in the unattended visual stream, highly salient inputs would induce an earlier response than less salient stimuli. While this model focuses on stimulus saliency, the OPR considers a lower level dimension of the stimulus, i.e., its intensity, and evaluates the probability of neural response to an incoming input, as presented in a random time point along the alpha phase. Therefore, the two proposals do not overlap and should be considered mutually exclusive; instead, they may be integrated to improve our understanding of the relationship between alpha oscillations, gamma activity, and visual perception.

Another theoretical strand has recently emphasized that alpha activity may be more closely related to a bias in the response and to variability in aspects of conscious perception, rather than to perceptual sensitivity. Specifically, lower levels of alpha activity have been associated with higher probabilities of positive responses, including in the absence of the stimulus, producing false alarms (Benwell et al., 2017; Iemi et al., 2017; Limbach and Corballis, 2016; Ruhnau et al., 2014). Conversely, in the OPR, the perceptual outcome is accounted for by neural activity in primary sensory areas and not by subsequent, higherorder processes. We postulate that the intriguing findings reported by Van Vugt and coworkers (2018) facilitate a reconciliation of the apparent inconsistencies between these recent interpretations and the OPR. First, the authors revealed that variability in the neural response to near-threshold stimuli is also present in the very first stages of the visual stream, with higher neural activity observed for hits than misses in V1. Moreover, the same study (van Vugt et al., 2018) also reported 
that false alarms are associated to neural activity in higher-order areas in the visual pathway, such as the dorso-lateral prefrontal cortex, suggesting that variability in perceptual outcome may also be due to subsequent processes in the visual stream. Notably, the false alarm rate in visual detection tasks has also been reported to be very low in studies on the effects of alpha activity on visual perception ( $~ 1.8 \%$; Busch and Van Rullen, 2010). Overall, decision bias is not likely to explain all of the effects of prestimulus alpha oscillations on perceptual outcome, and further studies are needed to provide insights into this issue. Currently, we propose that the OPR and theories that associate alpha activity with response bias should not be considered as alternatives, as alpha oscillations may affect perceptual outcome in several ways.

\section{Conclusions}

In summary, the hypothesis that the M/EEG signal in general, and neural oscillations in particular, may be related to more than a single mechanism has been already proposed (Cohen, 2017; Musall et al., 2014; Zoefel and VanRullen, 2017). However, the study of the relationship between macro- and meso-scale mechanisms, and particularly the link with behaviour, is still underinvestigated. While the OPR arises from the existing literature examining the effects of alpha oscillations on visual perception, it may also encourage a fruitful discussion about the relationship between neural activity across different scales, i.e., meso- and macro-scales, and behaviour by combining efforts from several methodological and theoretical perspectives.

\section{Funding}

This study was supported by the Italian Ministry of Health ('Ricerca Corrente').

\section{Appendix A. Supplementary data}

Supplementary material related to this article can be found, in the online version, at doi:https://doi.org/10.1016/j.neubiorev.2020.01. 037.

\section{References}

Ai, L., Ro, T., 2014. The phase of prestimulus alpha oscillations affects tactile perception. J. Neurophysiol. 111, 1300-1307. https://doi.org/10.1152/jn.00125.2013.

Arieli, A., Sterkin, A., Grinvald, A., Aertsen, A., 1996. Dynamics of ongoing activity: explanation of the large variability in evoked cortical responses. Science 273 (80), 1868-1871. https://doi.org/10.1126/science.273.5283.1868.

Bahramisharif, A., van Gerven, M.A.J., Aarnoutse, E.J., Mercier, M.R., Schwartz, T.H., Foxe, J.J., Ramsey, N.F., Jensen, O., 2013. Propagating neocortical gamma bursts are coordinated by traveling alpha waves. J. Neurosci. 33, 18849-18854. https://doi. org/10.1523/JNEUROSCI.2455-13.2013.

Baldassarre, A., Lewis, C.M., Committeri, G., Snyder, A.Z., Romani, G.L., Corbetta, M., 2012. Individual variability in functional connectivity predicts performance of a perceptual task. Proc. Natl. Acad. Sci. 109, 3516-3521. https://doi.org/10.1073/ pnas.1613915113.

Bastos, A.M., Schoffelen, J.-M., 2016. A tutorial review of functional connectivity analysis methods and their interpretational pitfalls. Front. Syst. Neurosci. 9, 1-23. https://doi. org/10.3389/fnsys.2015.00175.

Baumgarten, T.J., Schnitzler, A., Lange, J., 2016. Prestimulus alpha power influences tactile temporal perceptual discrimination and confidence in decisions. Cereb. Cortex 26, 891-903. https://doi.org/10.1093/cercor/bhu247.

Benwell, C.S.Y., Tagliabue, C.F., Veniero, D., Cecere, R., Savazzi, S., Thut, G., 2017. Prestimulus EEG power predicts conscious awareness but not objective visual performance. eNeuro 4, 1-17. https://doi.org/10.1523/ENEURO.0182-17.2017. e0182-17. 2017.

Berger, H., 1929. Über das elektrenkephalogramm des menschen. Arch. Psychiatr. Nervenkr. Z. Gesamte Neurol. Psychiatr. 87, 527-570. https://doi.org/10.1007/ BF01797193.

Bonnefond, M., Kastner, S., Jensen, O., 2017. Communication between brain areas based on nested oscillations. Eneuro 4, 1-14. https://doi.org/10.1523/ENEURO.0153-16. 2017. e0153-16.2017.

Bracci, E., Centonze, D., Bernardi, G., Calabresi, P., 2003. Voltage-dependent membrane potential oscillations of rat striatal fast-spiking interneurons. J. Physiol. 594, 121-130. https://doi.org/10.1113/jphysiol.2003.040857.

Britten, K.H., Shadlen, M.N., Newsome, W.T., Movshon, A.J., 1992. The analysis of visual motion: a comparison of neuronal and psychophysical performance. J. Neurosci. 12, 4745-4765.

Buia, C., Tiesinga, P., 2006. Attentional modulation of firing rate and synchrony in a model cortical network. J. Comput. Neurosci. 20, 247-264. https://doi.org/10.1007/ s10827-006-6358-0.

Busch, N.A., Van Rullen, R., 2010. Spontaneous EEG oscillations reveal periodic sampling of visual attention. Proc. Natl. Acad. Sci. 107, 16048-16053. https://doi.org/10. 1073/pnas.1004801107.

Busch, N.A., Dubois, J., Vanrullen, R., 2009. The phase of ongoing EEG oscillations predicts visual perception. J. Neurosci. 29, 7869-7876. https://doi.org/10.1523/ JNEUROSCI.0113-09.2009.

Buzsáki, G., Draguhn, A., 2004. Neuronal oscillations in cortical networks. Science 304 (80), 1926-1929.

Cameron, E.L., Tai, J.C., Carrasco, M., 2002. Covert attention affects the psychometric function of contrast sensitivity. Vision Res. 42, 949-967. https://doi.org/10.1016/ S0042-6989(02)00039-1.

Chaumon, M., Busch, N.A., 2014. Prestimulus neural oscillations inhibit visual perception via modulation of response gain. J. Cogn. Neurosci. 26, 2514-2529. https://doi.org/ 10.1162/jocn.

Cohen, M.X., 2017. Where does EEG come from and what does it mean? Trends Neurosci. 40, 208-218. https://doi.org/10.1016/j.tins.2017.02.004.

Cohen, M.X., Van Gaal, S., 2013. Dynamic interactions between large-scale brain networks predict behavioral adaptation after perceptual errors. Cereb. Cortex 23, 1061-1072. https://doi.org/10.1093/cercor/bhs069.

Donovan, I., Carrasco, M., 2018. Endogenous spatial attention during perceptual learning facilitates location transfer. J. Vis. 18, 1-16. https://doi.org/10.1167/18.11.7.

Dugué, L., Marque, P., VanRullen, R., 2011. The phase of ongoing oscillations mediates the causal relation between brain excitation and visual perception. J. Neurosci. 31, 11889-11893. https://doi.org/10.1523/JNEUROSCI.1161-11.2011.

Fiebelkorn, I.C., Pinsk, M.A., Kastner, S., 2018. A dynamic interplay within the frontoparietal network underlies rhythmic spatial attention. Neuron 99, 842-853. https:// doi.org/10.1016/j.neuron.2018.07.038. e8.

Frey, J.N., Ruhnau, P., Weisz, N., 2015. Not so different after all: the same oscillatory processes support different types of attention. Brain Res. 1626, 183-197. https://doi. org/10.1016/j.brainres.2015.02.017.

Fries, P., 2005. A mechanism for cognitive dynamics: neuronal communication through neuronal coherence. Trends Cogn. Sci. 9, 474-480. https://doi.org/10.1016/j.tics. 2005.08.011.

Fries, P., Reynolds, J.H., Rorie, A.E., Desimone, R., 2001. Modulation of oscillatory neuronal synchronization by selective visual attention. Science 291 (80), 1560-1563. https://doi.org/10.1126/science.1055465.

Fries, P., Nikolić, D., Singer, W., 2007. The gamma cycle. Trends Neurosci. 30, 309-316. https://doi.org/10.1016/j.tins.2007.05.005.

Haegens, S., Nacher, V., Luna, R., Romo, R., Jensen, O., 2011. $\alpha$-Oscillations in the monkey sensorimotor network influence discrimination performance by rhythmical inhibition of neuronal spiking. Proc. Natl. Acad. Sci. 108, 19377-19382. https://doi. org/10.1073/pnas.1117190108.

Haegens, S., Barczak, A., Musacchia, G., Lipton, M.L., Mehta, A.D., Lakatos, P., Schroeder, C.E., 2015. Laminar profile and physiology of the rhythm in primary visual, auditory, and somatosensory regions of neocortex. J. Neurosci. 35, 14341-14352. https://doi. org/10.1523/JNEUROSCI.0600-15.2015.

Hanslmayr, S., Aslan, A., Staudigl, T., Klimesch, W., Herrmann, C.S., Bäuml, K.H., 2007. Prestimulus oscillations predict visual perception performance between and within subjects. Neuroimage 37, 1465-1473. https://doi.org/10.1016/j.neuroimage.2007. 07.011.

Hara, Y., Pestilli, F., Gardner, J.L., 2014. Differing effects of attention in single-units and populations are well predicted by heterogeneous tuning and the normalization model of attention. Front. Comput. Neurosci. 8, 1-13. https://doi.org/10.3389/fncom. 2014.00012.

Harris, A.M., Dux, P.E., Mattingley, J.B., 2018. Detecting unattended stimuli depends on the phase of prestimulus neural oscillations. J. Neurosci. 38, 3092-3101. https://doi org /10.1523/JNEUROSCI.3006-17.2018.

Helfrich, R.F., Herrmann, C.S., Engel, A.K., Schneider, T.R., 2016. Different coupling modes mediate cortical cross-frequency interactions. Neuroimage 140, 76-82. https://doi.org/10.1016/j.neuroimage.2015.11.035.

Helfrich, R.F., Fiebelkorn, I.C., Szczepanski, S.M., Lin, J.J., Parvizi, J., Knight, R.T. Kastner, S., 2018. Neural mechanisms of sustained attention are rhythmic. Neuron 99, 854-865. https://doi.org/10.1016/j.neuron.2018.07.032. e5.

Henry, M.J., Obleser, J., 2012. Frequency modulation entrains slow neural oscillations and optimizes human listening behavior. Proc. Natl. Acad. Sci. 109, 20095-22100. https://doi.org/10.1073/pnas.1213390109.

Herrmann, K., Montaser-kouhsari, L., Carrasco, M., Heeger, D.J., 2010. When size matters : attention affects performance by contrast or response gain. Nat. Neurosci. 13, 1554-1559. https://doi.org/10.1038/nn.2669.

Hesselmann, G., Kell, Ca, Eger, E., Kleinschmidt, A., 2008a. Spontaneous local variations in ongoing neural activity bias perceptual decisions. Proc. Natl. Acad. Sci. 105, 10984-10989. https://doi.org/10.1073/pnas.0712043105.

Hyafil, A., Giraud, A.L., Fontolan, L., Gutkin, B., 2015. Neural cross-frequency coupling: connecting architectures, mechanisms, and functions. Trends Neurosci. 38, 725-740. https://doi.org/10.1016/j.tins.2015.09.001.

Iemi, L., Busch, N.A., 2018. Moment-to-Moment fluctuations in neuronal excitability Bias subjective perception rather than strategic decision-making. eNeuro ENEURO 2018https://doi.org/10.1523/ENEURO.0430-17.2018. 0430-17.

Iemi, L., Chaumon, M., Crouzet, S.M., Busch, N.A., 2017. Spontaneous neural oscillations Bias perception by modulating baseline excitability. J. Neurosci. 37, 807-819. https://doi.org/10.1523/JNEUROSCI.1432-16.2017. 
Jensen, O., Mazaheri, A., 2010. Shaping functional architecture by oscillatory alpha activity: gating by inhibition. Front. Hum. Neurosci. 4, 1-8. https://doi.org/10.3389/ fnhum. 2010.00186.

Jensen, O., Bonnefond, M., VanRullen, R., 2012. An oscillatory mechanism for prioritizing salient unattended stimuli. Trends Cogn. Sci. 16, 200-205. https://doi.org/10.1016/ j.tics.2012.03.002.

Jensen, O., Gips, B., Bergmann, T.O., Bonnefond, M., 2014. Temporal coding organized by coupled alpha and gamma oscillations prioritize visual processing. Trends Neurosci. 37, 357-369. https://doi.org/10.1016/j.tins.2014.04.001.

Jia, X., Kohn, A., 2011. Gamma rhythms in the brain. PLoS Biol. 9. https://doi.org/10. 1371/journal.pbio.1001045.

Kajikawa, Y., Schroeder, C.E., 2011. How local is the local field potential? Neuron 72, 847-858. https://doi.org/10.1016/j.neuron.2011.09.029.

Kayser, S.J., McNair, S.W., Kayser, C., 2016. Prestimulus influences on auditory perception from sensory representations and decision processes. Proc. Natl. Acad. Sci. 113, 4842-4847. https://doi.org/10.1073/pnas.1524087113.

Keitel, A., Gross, J., 2016. Individual human brain areas can be identified from their characteristic spectral activation fingerprints. PLoS Biol. 14, 1-22. https://doi.org/ 10.1371/journal.pbio.1002498.

Kim, Y.J., Grabowecky, M., Paller, K.A., Muthu, K., Suzuki, S., 2007. Attention induces synchronization-based response gain in steady-state visual evoked potentials. Nat. Neurosci. 10, 117-125. https://doi.org/10.1038/nn1821.

Klein, B.P., Harvey, B.M., Dumoulin, S.O., 2014. Attraction of position preference by spatial attention throughout human visual cortex. Neuron 84, 227-237. https://doi. org/10.1016/j.neuron.2014.08.047.

Klimesch, W., 2012. Alpha-band oscillations, attention, and controlled access to stored information. Trends Cogn. Sci. (Regul. Ed.) 16, 606-617. https://doi.org/10.1016/j. tics.2012.10.007.

Klimesch, W., Sauseng, P., Hanslmayr, S., 2007. EEG alpha oscillations: the inhibitiontiming hypothesis. Brain Res. Rev. 53, 63-88. https://doi.org/10.1016/j.brainresrev. 2006.06.003.

Landau, A.N., Fries, P., 2012. Attention samples stimuli rhythmically. Curr. Biol. 22, 1000-1004. https://doi.org/10.1016/j.cub.2012.03.054.

Landau, A.N., Schreyer, H.M., Van Pelt, S., Fries, P., 2015. Distributed attention is implemented through theta-rhythmic gamma modulation. Curr. Biol. 25, 2332-2337. https://doi.org/10.1016/j.cub.2015.07.048.

Lange, J., Keil, J., Schnitzler, A., van Dijk, H., Weisz, N., 2014. The role of alpha oscillations for illusory perception. Behav. Brain Res. 271, 294-301. https://doi.org/10. 1016/j.bbr.2014.06.015.

Leske, S., Ruhnau, P., Frey, J., Lithari, C., Müller, N., Hartmann, T., Weisz, N., 2015. Prestimulus network integration of auditory cortex predisposes near-threshold perception independently of local excitability. Cereb. Cortex 25, 4898-4907. https://doi org/10.1093/cercor/bhv212.

Limbach, K., Corballis, P.M., 2016. Prestimulus alpha power influences response criterion in a detection task. Psychophysiology 53, 1154-1164. https://doi.org/10.1111/psyp. 12666.

Ling, S., Carrasco, M., 2006. Sustained and transient covert attention enhance the signal via different contrast response functions. Vision Res. 46, 1210-1220. https://doi.org/ 10.1016/j.visres.2005.05.008.

Linkenkaer-Hansen, K., 2004. Prestimulus oscillations enhance psychophysical performance in humans. J. Neurosci. 24, 10186-10190. https://doi.org/10.1523/ JNEUROSCI.2584-04.2004.

Makeig, S., Westerfield, M., Jung, T.P., Enghoff, S., Towsend, J., Courchesne, E., Seijnowski, T.J., 2002. Dynamic brain sources of visual evoked responses. Science 295, 690-695.

Mathewson, K.E., Gratton, G., Fabiani, M., Beck, D.M., Ro, T., 2009. To see or not to see: prestimulus $\alpha$ phase predicts visual awareness. J. Neurosci. 29, 2725-2732. https:// doi.org/10.1523/JNEUROSCI.3963-08.2009s.

Mazaheri, A., Nieuwenhuis, I.L.C., Van Dijk, H., Jensen, O., 2009. Prestimulus alpha and $\mathrm{mu}$ activity predicts failure to inhibit motor responses. Hum. Brain Mapp. 30, 1791-1800. https://doi.org/10.1002/hbm.20763.

Musall, S., Von Pföstl, V., Rauch, A., Logothetis, N.K., Whittingstall, K., 2014. Effects of neural synchrony on surface EEG. Cereb. Cortex 24, 1045-1053. https://doi.org/10. 1093/cercor/bhs389.

Ni, J., Wunderle, T., Lewis, C.M., Desimone, R., Diester, I., Fries, P., 2016. Gammarhythmic gain modulation. Neuron 92, 240-251. https://doi.org/10.1016/j.neuron. 2016.09.003.

Osipova, D., Hermes, D., Jensen, O., 2008. Gamma power is phase-locked to posterior alpha activity. PLoS One 3, 1-7. https://doi.org/10.1371/journal.pone.0003990.

Palva, S., Palva, J.M., 2007. New vistas for $\alpha$-frequency band oscillations. Trends Neurosci. 30, 150-158. https://doi.org/10.1016/j.tins.2007.02.001.

Pernet, C.R., Sajda, P., Rousselet, G.A., 2011. Single-trial analyses: why bother? Front. Psychol. 2, 1-2. https://doi.org/10.3389/fpsyg.2011.00322.

Pestilli, F., Viera, G., Carrasco, M., 2007. How do attention and adaptation affect contrast sensitivity? J. Vis. 7 (9), 1-12. https://doi.org/10.1167/7.7.9.

Pestilli, F., Ling, S., Carrasco, M., 2009. A population-coding model of attention's influence on contrast response : estimating neural effects from psychophysical data. Vision Res. 49, 1144-1153. https://doi.org/10.1016/j.visres.2008.09.018.

Pham, A., Kiorpes, L., 2019. Endogenous attention improves perception in amblyopic macaques. J. Vis. 18, 1-13.

Pritchett, D.L., Siegle, J.H., Deister, C.A., Moore, C.I., 2015. For things needing your attention: the role of neocortical gamma in sensory perception. Curr. Opin. Neurobiol. 31, 254-263. https://doi.org/10.1016/j.conb.2015.02.004.

Ray, S., Maunsell, J.H.R., 2015. Do gamma oscillations play a role in cerebral cortex? Trends Cogn. Sci. 19, 78-85. https://doi.org/10.1016/j.tics.2014.12.002.

Reynolds, J.H., Chelazzi, L., 2004. Attentional modulation of visual processing. Annu.
Rev. Neurosci. 27, 611-647. https://doi.org/10.1146/annurev.neuro.26.041002. 131039.

Reynolds, J.H., Heeger, D.J., 2009. Review the normalization model of attention. Neuron 61, 168-185. https://doi.org/10.1016/j.neuron.2009.01.002.

Reynolds, J.H., Desimone, R., Reynolds, J.H., Pasternak, T., Desimone, R., York, N., 2000 Attention increases sensitivity of V4 neurons. Neuron 26, 703-714. https://doi.org/ 10.1016/S0896-6273(00)81206-4.

Romei, V., Brodbeck, V., Michel, C., Amedi, A., Pascual-Leone, A., Thut, G., 2008. Spontaneous fluctuations in posterior alpha-band EEG activity reflect variability in excitability of human visual areas. Cereb. Cortex 18, 2010-2018. https://doi.org/10. 1093/cercor/bhm229.

Romei, V., Gross, J., Thut, G., 2010. On the role of prestimulus alpha rhythms over occipito-parietal areas in visual input regulation: correlation or causation? J. Neurosci. 30, 8692-8697. https://doi.org/10.1523/JNEUROSCI.0160-10.2010.

Romei, V., Gross, J., Thut, G., 2012. Sounds reset rhythms of visual cortex and corresponding human visual perception. Curr. Biol. 22, 807-813. https://doi.org/10. 1016/j.cub.2012.03.025.

Roux, F., Wibral, M., Singer, W., Aru, J., Uhlhaas, P.J., 2013. The phase of thalamic alpha activity modulates cortical gamma-band activity: evidence from resting-state MEG recordings. J. Neurosci. 33, 17827-17835. https://doi.org/10.1523/JNEUROSCI. 5778-12.2013.

Ruhnau, P., Hauswald, A., Weisz, N., 2014. Investigating ongoing brain oscillations and their influence on conscious perception - network states and the window to consciousness. Front. Psychol. 5, 1-9. https://doi.org/10.3389/fpsyg.2014.01230.

Sadaghiani, S., Kleinschmidt, A., 2016. Brain networks and $\alpha$-Oscillations: structural and functional foundations of cognitive control. Trends Cogn. Sci. 20, 805-817. https:// doi.org/10.1016/j.tics.2016.09.004.

Sauseng, P., Klimesch, W., Stadler, W., Schabus, M., Doppelmayr, M., Hanslmayr, S., Gruber, W.R., Birbaumer, N., 2005. A shift of visual spatial attention is selectively associated with human EEG alpha activity. Eur. J. Neurosci. 22, 2917-2926. https:// doi.org/10.1111/j.1460-9568.2005.04482.x.

Schalk, G., 2015. A general framework for dynamic cortical function: the functionthrough-biased-oscillations (FBO) hypothesis. Front. Hum. Neurosci. 9, 1-10. https:// doi.org/10.3389/fnhum.2015.00352.

Schalk, G., Marple, J., Knight, R.T., Coon, W.G., 2017. Instantaneous voltage as an alternative to power- and phase-based interpretation of oscillatory brain activity. Neuroimage 157, 545-554. https://doi.org/10.1016/j.neuroimage.2017.06.014.

Schubert, R., Haufe, S., Blankenburg, F., Villringer, A., Curio, G., 2008. Now you'll feel itnow you won't: EEG rhythms predict the effectiveness of perceptual masking. J. Cogn. Neurosci. 2407-2419. https://doi.org/10.1162/jocn.2008.21174\r10.1162/ jocn.2008. 21174 [pii].

Sherman, M.T., Kanai, R., Seth, A.K., VanRullen, R., 2016. Rythmic influence of top-down perceptual priors in the phase of prestimulus occipital alpha oscillations. J. Cogn. Neurosci. 28, 1318-1330. https://doi.org/10.1162/jocn.

Song, K., Meng, M., Chen, L., Zhou, K., Luo, H., 2014. Behavioral oscillations in attention: rhythmic $\alpha$ pulses mediated through $\theta$ band. J. Neurosci. 34, 4837-4844. https://doi. org/10.1523/JNEUROSCI.4856-13.2014.

Spaak, E., Bonnefond, M., Maier, A., Leopold, D.A., Jensen, O., 2012. Layer-specific entrainment of gamma-band neural activity by the alpha rhythm in monkey visual cortex. Curr. Biol. 22, 2313-2318. https://doi.org/10.1016/j.cub.2012.10.020.

Spaak, E., de Lange, F.P., Jensen, O., 2014. Local entrainment of alpha oscillations by visual stimuli causes cyclic modulation of perception. J. Neurosci. 34, 3536-3544. https://doi.org/10.1523/JNEUROSCI.4385-13.2014.

Thut, G., Veniero, D., Romei, V., Miniussi, C., Schyns, P., Gross, J., 2011. Rhythmic TMS causes local entrainment of natural oscillatory signatures. Curr. Biol. 21, 1176-1185. https://doi.org/10.1016/j.cub.2011.05.049.

Thut, G., Bergmann, T.O., Fröhlich, F., Soekadar, S.R., Brittain, J.S., Valero-Cabré, A., Sack, A.T., Miniussi, C., Antal, A., Siebner, H.R., Ziemann, U., Herrmann, C.S., 2017. Guiding transcranial brain stimulation by EEG/MEG to interact with ongoing brain activity and associated functions: a position paper. Clin. Neurophysiol. 128, 843-857. https://doi.org/10.1016/j.clinph.2017.01.003.

Tiesinga, P.H.E., Fellous, J.M., Salinas, E., José, J.V., Sejnowski, T.J., 2004 Synchronization as a mechanism for attentional gain modulation. Neurocomputing 58-60, 641-646. https://doi.org/10.1016/j.neucom.2004.01.108.

van Boxtel, J.J.A., 2017. Different signal enhancement pathways of attention and consciousness underlie perception in humans. J. Neurosci. 37, 5912-5922. https://doi. org/10.1523/JNEUROSCI.1908-16.2017.

van Dijk, H., Schoffelen, J.M., Oostenveld, R., Jensen, O., 2008. Prestimulus oscillatory activity in the alpha band predicts visual discrimination ability. J. Neurosci. 28, 1816-1823. https://doi.org/10.1523/JNEUROSCI.1853-07.2008.

van Ede, F., Quinn, A.J., Woolrich, M.W., Nobre, A.C., 2018v. Neural oscillations: sustained rhythms or transient burst-events? Trends Neurosci. 41, 415-417. https://doi. org/10.1016/j.tins.2018.04.004.

van Es, M.W.J., Schoffelen, J.M., 2019v. Stimulus-induced gamma power predicts the amplitude of the subsequent visual evoked response. Neuroimage 186, 703-712. https://doi.org/10.1016/j.neuroimage.2018.11.029.

van Vugt, B., Dagnino, B., Vartak, D., Safaai, H., Panzeri, S., Dehaene, S., Roelfsema, P.R., 2018. The threshold for conscious report: signal loss and response bias in visual and frontal cortex. Science 360 (80), 537-542. https://doi.org/10.1126/science.aar7186.

VanRullen, R., Dubois, J., 2011. The psychophysics of brain rhythms. Front. Psychol. 2, 1-10. https://doi.org/10.3389/fpsyg.2011.00203.

VanRullen, R., Zoefel, B., Ilhan, B., 2014. On the cyclic nature of perception in vision versus audition. Philos. Trans. R. Soc. B Biol. Sci. 369https://doi.org/10.1098/rstb. 2013.0214. 20130214-20130214.

Vogels, R., Spileers, W., Orban, G.A., 1989. The response variability of striate cortical neurons in the behaving monkey. Exp. Brain Res. 77, 432-436. 
Wang, L., Krauzlis, R.J., 2018. Visual selective attention in mice. Curr. Biol. 28, 676-685. https://doi.org/10.1016/j.cub.2018.01.038. e4.

Weisz, N., Wühle, A., Monittola, G., Demarchi, G., Frey, J., Popov, T., Braun, C., 2014. Prestimulus oscillatory power and connectivity patterns predispose conscious somatosensory perception. Proc. Natl. Acad. Sci. 111, 417-425. https://doi.org/10.1073/ pnas.1317267111.

Whittingstall, K., Logothetis, N.K., 2009. Frequency-band coupling in surface EEG reflects spiking activity in monkey visual cortex. Neuron 64, 281-289. https://doi.org/10. 1016/j.neuron.2009.08.016.

Wichmann, F.A., Hill, N.J., 2001. The psychometric function: I. Fitting, sampling, and goodness-of-fit. Percept. Psychophys. 63, 1293-1313.

Williford, T., Maunsell, J.H.R., 2019. Effects of spatial attention on contrast response functions in macaque area V4. J. Neurophysiol. 96, 40-54. https://doi.org/10.1152/ jn.01207.2005.

Worden, M.S., Foxe, J.J., Wang, N., Simpson, G.V., 2000. Anticipatory biasing of visuospatial attention indexed by retinotopically specific alpha-band electroencephalography increases over occipital cortex. J. Neurosci. 20, 1-6 https:// doi.org/Rc63.

Wunderle, X.T., Eriksson, D., Peiker, C., Schmidt, X.E., 2015. Input and output gain modulation by the lateral interhemispheric network in early visual cortex. J. Neurosci. 35, 7682-7694. https://doi.org/10.1523/JNEUROSCI.4154-14.2015.

Zoefel, B., VanRullen, R., 2017. Oscillatory mechanisms of stimulus processing and selection in the visual and auditory systems: state-of-the-art, speculations and suggestions. Front. Neurosci. 11, 1-13. https://doi.org/10.3389/fnins.2017.00296. 